\title{
The effects of pre-habilitative conditioning on unloading-induced adaptations in young and aged neuromuscular systems
}

\author{
Michael R. Deschenes \\ William \& Mary, mrdesc@wm.edu \\ E. Grace Sherman \\ William \& Mary \\ Emily K. Glass \\ William \& Mary
}

Follow this and additional works at: https://scholarworks.wm.edu/aspubs

\section{Recommended Citation}

Deschenes, M. R., Sherman, E. G., \& Glass, E. K. (2012). The effects of pre-habilitative conditioning on unloading-induced adaptations in young and aged neuromuscular systems. Experimental gerontology, 47(9), 687-694. 


\title{
The effects of pre-habilitative conditioning on unloading-induced adaptations in young and aged neuromuscular systems
}

\author{
Michael R. Deschenes a,b,*, E. Grace Sherman a , Emily K. Glass ${ }^{\text {a,b }}$ \\ a Department of Kinesiology and Health Sciences, The College of William \& Mary, Williamsburg, VA 23187-8795, USA \\ ${ }^{\mathrm{b}}$ Program in Neuroscience, The College of William \& Mary, Williamsburg, VA 23187-8795, USA
}

\section{A R T I C L E I N F O}

\section{Article history:}

Received 7 June 2012

Accepted 19 June 2012

Available online 28 June 2012

Section Editor: Christiaan Leeuwenburgh

\section{Keywords:}

Myofiber

Synapse

Atrophy

Disuse

Old

Unweighting

\begin{abstract}
A B S T R A C T
The capacity of pre-habilitative conditioning - exercise performed a priori - to mitigate neuromuscular maladaptations to disuse is unclear. This study evaluated pre-habilitation by examining neuromuscular junctions (NMJs) and the myofibers they innervate in young adult and aged muscles. Within each age category, 40 rats were divided into four treatment groups: 1) control, 2) hindlimb suspended (unloaded), 3) prehabilitative conditioning preceding hindlimb suspension, and 4) pre-habilitative conditioning alone. Cytofluorescent staining was used to visualize NMJs, and histochemical staining to assess myofiber profiles (size and type). Statistical analysis featured 2-way ANOVA with main effects for age and treatment, along with interaction. NMJs consistently revealed significant $(P \leq 0.05)$ main effects for age, but not treatment, or interaction. Typically, aged NMJs showed elongated nerve terminal branching, and more dispersed post-synaptic clusters of ACh receptors, resulting in reduced post-synaptic area per given length of pre-synaptic branching. Analysis of myofiber profiles showed significant main effects for age, treatment, and their interaction. Aged myofibers were smaller than the young ones and a higher percentage of them were Type I. Aged fibers experienced significantly greater unloading-induced atrophy than the young ones. Pre-habilitative conditioning significantly attenuated unloading-induced atrophy among aged, but not young myofibers. It was also observed that pre-habilitative conditioning alone increased myofiber size among aged, but not young adult muscles. In summary, myofibers were more sensitive than NMJs to the treatment interventions implemented. Although more sensitive to the negative effects of muscle unloading, aged myofibers were also more responsive to the hypertrophic effects of pre-habilitative conditioning.
\end{abstract}

(c) 2012 Elsevier Inc. All rights reserved.

\section{Introduction}

Muscle unloading elicits a number of detrimental effects of the neuromuscular system. These typically include myofiber atrophy and strength declines, myofiber type conversions and decrements in muscular power (Adams et al., 2003; Caiozzo et al., 1995; Fitts et al., 2000; Kraemer et al., 2000; Musacchia et al., 1990), as well as remodeling of the neuromuscular junction, the vital synapse enabling communication between motor neurons and their associated myofibers (Deschenes et al., 2005). More recently it has been documented that aging influences those modifications in that, compared to young ones, aged neuromuscular systems experience greater disturbances in neuromuscular function and structure following periods of muscle unloading (Deschenes and Wilson, 2003). Since the pioneering work of DeLorme and Watkins (1948) and DeLorme et al. (1950), rehabilitative conditioning programs (i.e. exercise training conducted following muscle unloading) have been used to effectively expedite the

\footnotetext{
* Corresponding author at: Department of Kinesiology and Health Sciences, The College of William \& Mary, Williamsburg, VA 23187-8795, USA. Tel.: +1 757221 2778; fax: +1 7572212761 .

E-mail address: mrdesc@wm.edu (M.R. Deschenes).
}

rate of recovery of the neuromuscular system from negative adaptations to muscle disuse. However, the potential of prehabilitative conditioning (i.e. exercise training performed prior to the onset of muscle unloading) to mitigate, or even prevent, the neuromuscular maladaptations related to muscle unloading remains largely unexplored. This is particularly true of the aged population which, unfortunately, is more likely to be subjected to muscle unloading due to a greater incidence of accidental falls, joint replacement surgery, etc. (Lavernia et al., 2006; Lipsitz et al., 1994). Given this dearth of information, the investigation reported here aimed to: 1) assess the efficacy of prehabilitative conditioning in moderating unloading-induced perturbations of myofiber profiles and neuromuscular junction morphology, and 2) determine whether the impact of the prehabilitative exercise regimen would differ between the neuromuscular systems of young and aged individuals.

\section{Materials and methods}

\subsection{Subjects}

Male Fischer 344 rats, purchased from the National Institutes on Aging Colonies, served as subjects. Forty young adult ( 9 month old), 
and 40 aged (25 month old) rats were randomly assigned to four treatment groups within each age category: 1) control (CTL), 2) hindlimb suspension (HS), 3) prehabilitative conditioning followed by hindlimb suspension ( $\mathrm{PH}-\mathrm{HS}$ ), and 4) prehabilitative conditioning by itself $(\mathrm{PH})$. In total then, eight treatment groups were formed with $\mathrm{N}=10$ for each group. It is important to note that based upon their average life span, male Fischer 344 rats at 9 and 25 months of age are the age equivalent, respectively, of 26 and 74 year old human males (Arias, 2006; Turturro et al., 1999).

All animals were provided with standard rat chow and water on an ad libitum basis. For those animals undergoing hindlimb suspension, the chow pellets were ground up with an ice crusher to make the handling/eating of them easier. Rats were housed in a constant 21-22 ${ }^{\circ} \mathrm{C}$ environment with a $12-12 \mathrm{~h}$ light-dark cycle.

\subsection{Treatment protocols}

The hindlimb suspension model first described by Morey (1979) was used to evoke a state of unloading in the hindlimb muscles. In this model, the animal's hindfeet are elevated just enough to prevent weight bearing activity. This is achieved by placing an adhesive strip along two sides of the tail with a clip attached so that the clip can be placed into a swivel device that is secured above the rat which, in turn, allows the rat to move in a $360^{\circ}$ arc using its forelimbs. Animals undergoing this intervention remained in this position $24 \mathrm{~h}$ a day for 14 consecutive days.

The prehabilitative conditioning program consisted of treadmill running 5 days a week for a two week period. At the start of the regimen, rats ran for $15 \mathrm{~min}$ at a velocity of $7.5 \mathrm{~m} / \mathrm{min}$ at a $0^{\circ}$ grade. The duration and velocity of the running sessions were gradually increased so that by the end of the two week intervention rats were running for $30 \mathrm{~min}$ at $9.5 \mathrm{~m} / \mathrm{min}$. Both the aged and the young adult rats completed the same training regimen. In the $\mathrm{PH}-\mathrm{HS}$ groups (young adult and aged) animals were then subjected -within a 48 hour period - to the hindlimb suspension treatment. But the $\mathrm{PH}$ groups were euthanized before participating in the muscle unloading intervention. Similarly, those animals assigned to the HS groups were placed in the muscle unloading conditions without initially completing the program of prehabilitative conditioning. Finally, the CTL groups simply remained in their housing tubs and were allowed to carry out normal weight bearing and ambulatory activity. All procedures used in the treatment and care of animals were approved by the animal use and care committee of the College of William \& Mary which operates in accordance with the National Institutes of Health Guide for the Care and Use of Laboratory Animals as revised in 1996.

\subsection{Tissue preparation and storage}

At the conclusion of the treatment period, animals were euthanized and soleus muscles were dissected out, cleared of fat and connective tissue and quickly frozen at a resting length in isopentane chilled with liquid nitrogen. Muscles were then stored at $-85{ }^{\circ} \mathrm{C}$ until analysis. The soleus was selected for study because as the principal postural muscle (Roy et al., 1991), it is keenly sensitive to the model of muscle unloading employed here. Furthermore, the soleus is heavily recruited during locomotor activity and as a result, it is also affected by the treadmill running program used for prehabilitative conditioning (Roy et al., 1985).

\subsection{Cytofluorescent staining of NMJs}

To visualize NMJs, $50 \mu \mathrm{m}$ thick longitudinal sections of the middle one-third of the muscle were obtained at $-20{ }^{\circ} \mathrm{C}$ on a cryostat (Cryocut 1800; Reichert-Jung, NuBloch, Germany). To prevent contraction of sections, microscope slides were pretreated in a $3 \%$ EDTA solution as previously described (Pearson and Sabarra, 1974).
Sections were washed $4 \times 15 \mathrm{~min}$ in a phosphate buffered saline (PBS) containing $1 \%$ bovine serum albumin (BSA). Sections were then incubated in a humidified chamber overnight at $4{ }^{\circ} \mathrm{C}$ in a supernatant of the primary antibody RT97 (Developmental Studies Hybridoma Bank, University of Iowa), diluted 1:20 in PBS with $1 \%$ BSA. The RT97 antibody reacts with non-myelinated segments of pre-synaptic nerve terminals (Anderton et al., 1982). The next day, sections were washed $4 \times 15$ in PBS with $1 \%$ BSA before incubating for $2 \mathrm{~h}$ at room temperature in fluorescein isothiocyanate (FITC) conjugated secondary immunoglobulin (Sigma Chemical, St. Louis, MO) that was diluted 1:150 in PBS with 1\% BSA. Sections were then washed $4 \times 15$ min in PBS with $1 \%$ BSA. Following this, sections were incubated in a humidified chamber overnight at $4{ }^{\circ} \mathrm{C}$ in a solution containing rhodamine conjugated $\alpha$-bungarotoxin (BTX; Molecular Probes, Eugene, OR) diluted 1:600 in PBS along with an anti-slow myosin heavy chain ascites fluid (Sigma Chemical, St. Louis, MO) diluted 1:40. BTX recognizes post-synaptic acetylcholine $(\mathrm{ACh})$ receptors, while the anti-slow immunogen enabled us to determine whether the endplate resided on a fast- or slow-twitch myofiber. The next day, sections were washed $4 \times 15 \mathrm{~min}$ in PBS with $1 \%$ BSA before incubating them for $1 \mathrm{~h}$ at room temperature in AlexaFluor 647 (Molecular Probes, Eugene, OR) labeled secondary antibody to bind with the anti-slow primary antibody. Sections were given a final wash $(4 \times 15 \mathrm{~min})$ before being lightly coated with Pro Long (Molecular probes, Eugene, OR) and having cover slips applied. Slides were then coded with respect to treatment group to allow for blinded evaluation of NMJ morphology and then stored at $-20{ }^{\circ} \mathrm{C}$ until analysis. An example of this cytofluorescent staining of pre- and post-synaptic components of the NMJ is displayed in Fig. 1.

Pre-synaptic variables of NMJs assessed included: 1) the number of branches identified at the nerve terminal, 2) the total length of those branches, 3) average length per branch, and 4) branching complexity which, as described by Tomas et al. (1990) is derived by multiplying the number of branches by the total length of those branches and dividing that figure by 100 . Post-synaptic variables of interest included: 1 ) the total perimeter, or the length encompassing the entire endplate composed of stained receptor clusters and non-stained regions interspersed within those clusters, 2) stained perimeter, or the composite length of tracings around individual receptor clusters, 3 ) total area, which includes stained receptors along with non-stained regions interspersed among receptor clusters, 4) stained area, or the cumulative areas occupied by ACh receptor clusters, and 5) dispersion of endplates, which was assessed by dividing the endplate's stained area by its total area and multiplying by 100. In this study, preto post-synaptic coupling was quantified by dividing the NMJ's post-synaptic stained area by its total length of nerve terminal branching.

\subsection{Histochemical staining of myofibers}

To assess myofiber profiles, $10 \mu \mathrm{m}$ thick transverse sections were taken from the midbelly of the contralateral muscle using a cryostat set at $-20{ }^{\circ} \mathrm{C}$. Sections were stained for myofibrillar ATPase activity following pre-incubation at $\mathrm{pH} 4.55$ according to Nemeth and Pette (1981). A random sample of 125-150 myofibers from each muscle was used to determine the average myofiber size (i.e., cross-sectional area) and fiber type composition for that muscle. Fiber types were classified as either Type I, IIA, or IIX. It has been documented that the soleus muscle of the rat does not express Type IIB fibers (Delp and Duan, 1996). Slides were coded with respect to treatment group to prevent bias during measurement.

\subsection{Microscopy}

An Olympus Fluoview FV 300 confocal system featuring three lasers and an Olympus BX60 fluorescent microscope (Olympus America, Melville, NY) was used to collect images of NMJs and to determine whether they were located on slow- or fast-twitch 

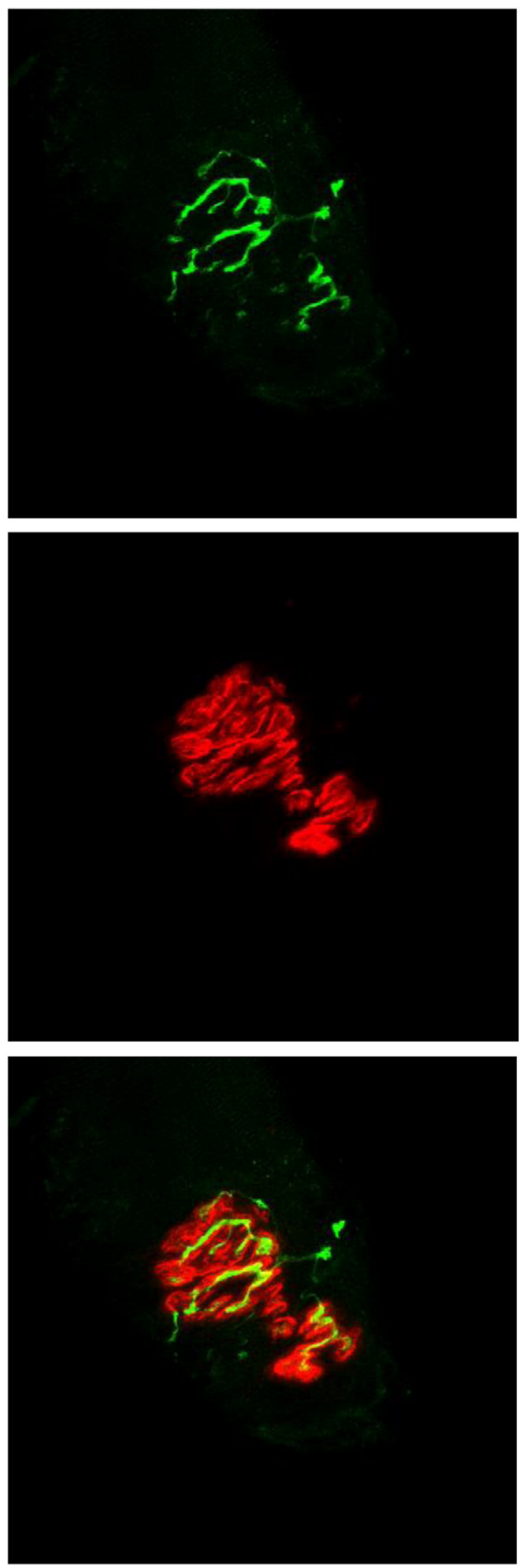

Fig. 1. Representative fluorescent staining of the neuromuscular junction. Pre-synaptic nerve terminal branches are depicted in the top frame. In the middle frame, post-synaptic acetylcholine receptors are seen. The bottom panel shows the overlap between pre-synaptic nerve terminal branches, stained green with the fluorochrome fluorescein, and post-synaptic acetylcholine receptors stained red with the fluorochrome rhodamine. Original magnification of $1000 \times$. myofibers. Using a $100 \times$ oil immersion objective, it was initially established that the entire NMJ was within the longitudinal borders of the myofiber and that damage to the structure had not occurred during sectioning. A detailed image of the entire NMJ was constructed from a z-series of scans taken at $1 \mu \mathrm{m}$ thick increments. To determine whether the NMJ resided on a fast- or a slow-twitch myofiber, a single scan was collected of the fiber using the appropriate wavelength to detect the AlexaFluor 647 fluorochrome. Digitized, two-dimensional images of NMJs were stored on the system's hard drive and later quantified with the Image-Pro Plus software (Media Cybernetics, Silver Spring, MD). In slow-twitch myofibers - which are dominant within the soleus - 10-12 NMJs were quantified and measurements were averaged to represent slow-twitch NMJs within that muscle. But in the soleus fast-twitch myofibers are far more sparse so measurements from a minimum of 5 NMJs were averaged together to represent fast-twitch NMJs of that muscle.

To assess myofiber profiles of each soleus muscle, an Olympus BX41 phase contrast microscope fitted with a $40 \times$ objective was used. Myofiber cross-sectional areas were quantified with the Image-Pro Express software. A random sample of 125-150 myofibers from each muscle was analyzed to determine the average myofiber size (i.e., cross-sectional area) and fiber type composition for that muscle.

\subsection{Statistical analysis}

Data are reported as means \pm SE. Pre- to post-intervention body mass data were compared with dependent $t$-tests. In addition, independent $t$-tests were used to compare the body mass of young adult vs. aged animals both before and after the treatment periods. All other variables were assessed with 2-way ANOVA with main effects for treatment and age. In the event of a significant F-ratio, for either the main effect or interaction, a post-hoc analysis was conducted to identify pair-wise differences. In all cases, significance was established at $\mathrm{P} \leq 0.05$.

\section{Results}

\subsection{Body mass}

The impact of the four intervention protocols on the body mass of young adult and aged rats is displayed in Table 1. In examining the effect of aging alone on body mass, there was virtually no difference in the pre-intervention body mass between the young and aged control animals (396 vs. $398 \mathrm{~g}$, respectively). Statistical analysis revealed, however, that among young rats a significant difference in pre- to post-intervention body mass occurred among all four treatment groups. But while the HS and the HS-PH groups experienced declines in body mass as a result of their interventions, the control and $\mathrm{PH}$ groups showed increases over that same time frame. Moreover, when comparing the body mass of the four treatment groups it was determined that at the post-, but not the pre-intervention time point there were significant differences between all four groups of young animals (i.e., no two groups were the same).

In contrast to young rats where it was observed that the various interventions significantly affected the body mass of all four treatment groups, among the aged only the HS and $\mathrm{PH}-\mathrm{HS}$ groups experienced significant pre- to post-intervention changes in body mass, and both groups displayed a significant decrease. And although there were no between treatment group differences noted among aged rats at pre-intervention, it was found that CTL and PH rats were significantly heavier than the HS and PH-HS animals at post-intervention.

\subsection{Neuromuscular junctions}

Synapses located on slow-twitch myofibers and those identified on fast-twitch myofibers were analyzed separately and results generated 
Table 1

Effects of hindlimb suspension (HS), hindlimb suspension preceded by pre-habilitation (PH-HS), and pre-habilitation by itself (HS) on body mass ( $\mathrm{g}$ ) of young and aged rats.

\begin{tabular}{lllll}
\hline & Control & HS & PH-HS & PH \\
\hline $\begin{array}{l}\text { Young } \\
\text { Pre-intervention }\end{array}$ & $395.7 \pm 4.2$ & $394.3 \pm 4.8$ & $361.3 \pm 7.3$ & $375.6 \pm 6.4$ \\
Post-intervention & $456.6 \pm 5.7^{\mathrm{a}}$ & $345.5 \pm 6.5^{\mathrm{abcd}}$ & $321.7 \pm 7.2^{\mathrm{abc}}$ & $393.2 \pm 6.8^{\mathrm{ab}}$ \\
& & & & \\
Aged & & & & \\
Pre-intervention & $397.9 \pm 10.8$ & $396.0 \pm 12.2$ & $404.1 \pm 7.8$ & $412.4 \pm 12.6$ \\
Post-intervention & $402.4 \pm 13.5$ & $369.6 \pm 14.5^{\mathrm{abc}}$ & $361.1 \pm 6.2^{\mathrm{abc}}$ & $419.6 \pm 11.1$ \\
\hline
\end{tabular}

Values are means $\pm S E, N=10$ /group.

a Indicates significant $(\mathrm{P} \leq 0.05)$ pre- to post-intervention difference in the same age category.

$\mathrm{b}$ Indicates significant $(\mathrm{P} \leq 0.05)$ difference from the post-intervention control group in the same age category.

${ }^{c}$ Indicates significant $(\mathrm{P} \leq 0.05)$ difference from the post-intervention $\mathrm{PH}$ group in the same age category.

${ }^{\mathrm{d}}$ Indicates significant $(\mathrm{P} \leq 0.05)$ difference from the post-intervention $\mathrm{PH}-\mathrm{HS}$ group in the same age category.

can be viewed in Tables 2 and 3, respectively. Among the predominant slow-twitch NMJs - recall that the majority fiber type in the soleus is slow-twitch, or Type I (Armstrong and Phelps, 1984; Delp and Duan, 1996) - a significant main effect of age was revealed in the pre-synaptic nerve terminal branch number, total branch length, and branching complexity (aged > young). The average branch length, however, was similar between the two age categories. In contrast to the findings on the effect of aging on the nerve terminal morphology, in not a single instance was a significant main effect of treatment identified, nor was there a significant interactive effect for any of those variables.

Regarding the post-synaptic endplate morphology of slow-twitch NMJs, no significant main effects, or interactive effects were identified when examining the total perimeter length, stained perimeter length, or total area occupied by ACh receptors. In contrast, our analysis of post-synaptic stained area, dispersion of receptors, as well as pre- to post-synaptic coupling revealed a significant main effect of age. But unlike the nerve terminal branching where aging was associated with increased length, aged endplates were found to be smaller, with receptor clusters more dispersed, than the young ones. And when linked with expanded terminal branch length, the smaller post-synaptic stained areas among aged endplates resulted in a significantly reduced ratio of endplate size relative to nerve terminal branching (pre- to post-synaptic coupling) among aged NMJs. These age-related post-synaptic effects were consistent as the aged from each treatment group were found to significantly differ from the young of the same treatment group.

In examining ANOVA results from NMJs of fast-twitch myofibers, it appeared that the remodeling was similar, yet more pervasive than what was observed in slow-twitch NMJs. Again, it was demonstrated that significant effects for aging, but not treatment or interaction, had occurred. But unlike in slow-twitch NMJs where some, but not all of the variables assessed displayed the effects of aging, in fast-twitch NMJs each of those variables - both pre- and post-synaptic - showed a significant main effect of aging. With respect to pre-synaptic nerve terminal branching, it was determined that as with slow-twitch NMJs, branching measures were significantly expanded among the aged fast-twitch synapses. This was true of each pre-synaptic parameter assessed; branch number, total branch length, average branch length, and branching complexity.

Unlike what was revealed among slow-twitch endplates, however, aging significantly affected the total perimeter and stained perimeter lengths surrounding ACh receptor clusters, as well as the total area of the endplate region. More specifically, it was noted that in those variables older animals exhibited expanded dimensions relative to young adults. But fast-twitch endplates showed the same results for the remaining post-synaptic parameters that were detected among slow-twitch synapses. That is, stained areas were greater among young endplates than the aged ones, with dispersion of receptor clusters being more pronounced among the aged, while the relative area of ACh receptors innervated per given length of pre-synaptic nerve terminal branching was also greater in young compared to aged NMJs. And although it was once again found that only significant main effects of age - not treatment or interaction - were detected among fast-twitch NMJs, each of the morphological variables assessed exhibited an effect of age in those fast-twitch synapses. This compared with slow-twitch NMJs where only six of the ten parameters quantified revealed a significant main effect of age.

\subsection{Myofiber profiles}

Our statistical analysis of myofiber profiles of soleus muscles showed that myofibers underwent a greater degree of remodeling than the

Table 2

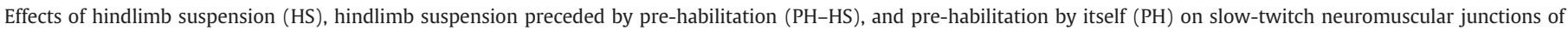
young and aged soleus muscles.

\begin{tabular}{|c|c|c|c|c|c|c|c|c|}
\hline & \multicolumn{2}{|l|}{ Control } & \multicolumn{2}{|l|}{ HS } & \multicolumn{2}{|l|}{ PH-HS } & \multicolumn{2}{|l|}{$\mathrm{PH}$} \\
\hline & Young & Aged & Young & Aged & Young & Aged & Young & Aged \\
\hline \multicolumn{9}{|c|}{ Pre-synaptic nerve terminal branching } \\
\hline Branch number ${ }^{\mathrm{a}}$ & $10.5 \pm 0.4$ & $11.3 \pm 1.1^{\mathrm{b}}$ & $9.1 \pm 0.5$ & $9.9 \pm 0.8$ & $8.7 \pm 0.4$ & $10.2 \pm 1.0$ & $8.8 \pm 0.6$ & $10.7 \pm 0.4$ \\
\hline Total branch length $(\mu \mathrm{m})^{\mathrm{a}}$ & $186 \pm 11$ & $206 \pm 16$ & $166 \pm 9^{c}$ & $210 \pm 12$ & $181 \pm 16$ & $212 \pm 19^{d}$ & $172 \pm 12$ & $206 \pm 11$ \\
\hline Mean branch length $(\mu \mathrm{m})$ & $19 \pm 1$ & $19 \pm 1$ & $20 \pm 1$ & $23 \pm 2$ & $22 \pm 2$ & $23 \pm 2$ & $21 \pm 1$ & $20 \pm 1$ \\
\hline Branching complexity $^{\mathrm{a}}$ & $21.6 \pm 1.6$ & $28.0 \pm 5.0^{\mathrm{b}}$ & $17.4 \pm 1.6$ & $24.7 \pm 3.5$ & $17.6 \pm 2.0$ & $25.1 \pm 4.6$ & $17.1 \pm 2.1$ & $25.4 \pm 1.6$ \\
\hline \multicolumn{9}{|l|}{ Post-synaptic endplate } \\
\hline Total perimeter $(\mu \mathrm{m})$ & $191 \pm 12$ & $188 \pm 8$ & $183 \pm 8$ & $208 \pm 12$ & $174 \pm 5$ & $197 \pm 10$ & $199 \pm 14$ & $180 \pm 6$ \\
\hline Stained perimeter $(\mu \mathrm{m})$ & $358 \pm 21$ & $335 \pm 25$ & $321 \pm 19$ & $354 \pm 27$ & $323 \pm 17$ & $327 \pm 18$ & $328 \pm 19$ & $301 \pm 19$ \\
\hline Total area $\left(\mu \mathrm{m}^{2}\right)$ & $1070 \pm 99$ & $1141 \pm 73$ & $980 \pm 53$ & $1063 \pm 102$ & $951 \pm 64$ & $1065 \pm 69$ & $999 \pm 48$ & $1053 \pm 96$ \\
\hline Stained area $\left(\mu \mathrm{m}^{2}\right)^{\mathrm{a}}$ & $542 \pm 53$ & $361 \pm 50^{\mathrm{e}}$ & $511 \pm 27$ & $357 \pm 37^{\mathrm{e}}$ & $481 \pm 29$ & $368 \pm 24^{\mathrm{e}}$ & $538 \pm 30$ & $383 \pm 32^{\mathrm{e}}$ \\
\hline Dispersion $(\%)^{\mathrm{a}}$ & $54.2 \pm 4.0$ & $35.0 \pm 2.6^{\mathrm{e}}$ & $52.9 \pm 2.1$ & $38.7 \pm 5.1^{\mathrm{e}}$ & $52.0 \pm 1.6$ & $38.8 \pm 3.2^{\mathrm{e}}$ & $57.6 \pm 3.8$ & $39.6 \pm 3.5^{\mathrm{e}}$ \\
\hline Pre- to post-synaptic coupling ${ }^{\mathrm{a}}$ & $3.0 \pm 0.2$ & $2.0 \pm 0.2^{\mathrm{e}}$ & $3.5 \pm 0.2$ & $2.0 \pm 0.2^{\mathrm{e}}$ & $3.1 \pm 0.3$ & $2.2 \pm 0.2^{\mathrm{e}}$ & $3.5 \pm 0.3$ & $2.1 \pm 0.1^{\mathrm{e}}$ \\
\hline
\end{tabular}

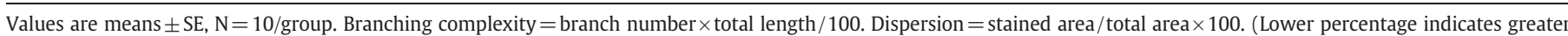
dispersion.) Pre- to post-synaptic coupling = endplate stained area/total nerve terminal branch length.

a Indicates significant $(\mathrm{P} \leq 0.05)$ main effect of age.

b Indicates significant $(\mathrm{P} \leq 0.05)$ difference from young $\mathrm{HS}$, young $\mathrm{PH}-\mathrm{HS}$, and young $\mathrm{PH}$ groups.

c Indicates significant $(\mathrm{P} \leq 0.05)$ difference from all four aged treatment groups.

d Indicates significant $(\mathrm{P} \leq 0.05)$ difference from young $\mathrm{PH}$.

e Indicates significant $(\mathrm{P} \leq 0.05)$ difference from all four young treatment groups. 
Table 3

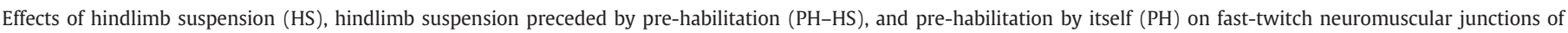
young and aged soleus muscles.

\begin{tabular}{|c|c|c|c|c|c|c|c|c|}
\hline & \multicolumn{2}{|l|}{ Control } & \multicolumn{2}{|l|}{ HS } & \multicolumn{2}{|l|}{ PH-HS } & \multicolumn{2}{|l|}{$\mathrm{PH}$} \\
\hline & Young & Aged & Young & Aged & Young & Aged & Young & Aged \\
\hline \multicolumn{9}{|c|}{ Pre-synaptic nerve terminal branching } \\
\hline Branch number ${ }^{\mathrm{a}}$ & $9.9 \pm 0.8$ & $9.8 \pm 0.7$ & $7.9 \pm 1.0$ & $10.7 \pm 1.1^{\mathrm{b}}$ & $8.1 \pm 0.9$ & $9.7 \pm 0.7$ & $7.9 \pm 1.0$ & $9.8 \pm 0.8$ \\
\hline Total branch length $(\mu \mathrm{m})^{\mathrm{a}}$ & $172 \pm 14$ & $208 \pm 20^{b}$ & $146 \pm 10$ & $249 \pm 38^{c}$ & $134 \pm 13$ & $199 \pm 20^{b}$ & $144 \pm 15$ & $185 \pm 10$ \\
\hline Mean branch length $(\mu \mathrm{m})^{\mathrm{a}}$ & $19 \pm 2$ & $22 \pm 1$ & $21 \pm 2$ & $22 \pm 2$ & $18 \pm 1$ & $26 \pm 6^{\mathrm{d}}$ & $20 \pm 2$ & $21 \pm 2$ \\
\hline Branching complexity ${ }^{\mathrm{a}}$ & $18.2 \pm 2.7$ & $24.7 \pm 4.3^{\mathrm{b}}$ & $12.0 \pm 2.3$ & $35.0 \pm 9.2^{\mathrm{c}}$ & $12.3 \pm 2.2$ & $22.7 \pm 3.4$ & $13.3 \pm 2.5$ & $20.2 \pm 2.6$ \\
\hline \multicolumn{9}{|l|}{ Post-synaptic endplate } \\
\hline Total perimeter $(\mu \mathrm{m})^{\mathrm{a}}$ & $153 \pm 8$ & $189 \pm 10^{\mathrm{e}}$ & $167 \pm 5$ & $195 \pm 7^{c}$ & $153 \pm 13$ & $188 \pm 13^{\mathrm{e}}$ & $150 \pm 11$ & $166 \pm 9$ \\
\hline Stained perimeter $(\mu \mathrm{m})^{\mathrm{a}}$ & $266 \pm 24$ & $324 \pm 36$ & $270 \pm 20$ & $336 \pm 25$ & $250 \pm 30$ & $359 \pm 41^{c}$ & $235 \pm 22^{f}$ & $294 \pm 30$ \\
\hline Total area $\left(\mu \mathrm{m}^{2}\right)^{\mathrm{a}}$ & $803 \pm 57$ & $1082 \pm 140^{g}$ & $833 \pm 64$ & $1114 \pm 114^{\mathrm{e}}$ & $761 \pm 57$ & $1114 \pm 148^{\mathrm{c}}$ & $764 \pm 82$ & $939 \pm 94$ \\
\hline Stained area $\left(\mu \mathrm{m}^{2}\right)^{\mathrm{a}}$ & $449 \pm 35$ & $353 \pm 30^{\mathrm{h}}$ & $490 \pm 49$ & $417 \pm 64$ & $424 \pm 28$ & $403 \pm 50$ & $426 \pm 45$ & $374 \pm 39$ \\
\hline Dispersion $(\%)^{\mathrm{a}}$ & $56.9 \pm 2.4$ & $37.5 \pm 3.1^{\mathrm{c}}$ & $59.6 \pm 1.9$ & $39.7 \pm 2.4^{\mathrm{c}}$ & $56.6 \pm 3.4$ & $38.9 \pm 3.7^{c}$ & $57.3 \pm 3.3$ & $42.1 \pm 2.3^{\mathrm{c}}$ \\
\hline Pre- to post-synaptic coupling ${ }^{\mathrm{a}}$ & $2.8 \pm 0.2$ & $1.9 \pm 0.2^{\mathrm{C}}$ & $3.8 \pm 0.5$ & $2.1 \pm 0.2^{\mathrm{b}}$ & $3.5 \pm 0.4$ & $2.5 \pm 0.2^{b}$ & $3.7 \pm 0.5$ & $2.1 \pm 0.2^{\mathrm{c}}$ \\
\hline
\end{tabular}

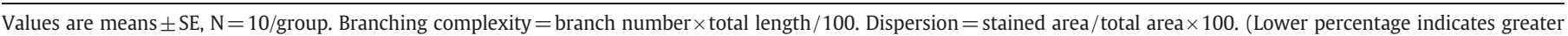
dispersion.) Pre- to post-synaptic coupling = endplate stained area/total nerve terminal branch length.

${ }^{a}$ Indicates significant $(\mathrm{P} \leq 0.05)$ main effect of age.

b Indicates significant $(\mathrm{P} \leq 0.05)$ difference from young $\mathrm{HS}$, young $\mathrm{PH}-\mathrm{HS}$, and young $\mathrm{PH}$ groups.

c Indicates significant $(\mathrm{P} \leq 0.05)$ difference from all four young treatment groups.

d Indicates significant $(\mathrm{P} \leq 0.05)$ difference from young control and young $\mathrm{PH}-\mathrm{HS}$ groups.

e Indicates significant $(\mathrm{P} \leq 0.05)$ difference from young control, young $\mathrm{PH}-\mathrm{HS}$, and young $\mathrm{PH}$ groups.

${ }^{f}$ Indicates significant $(\mathrm{P} \leq 0.05)$ difference from aged control, and aged HS groups.

g Indicates significant $(\mathrm{P} \leq 0.05)$ difference from young $\mathrm{PH}-\mathrm{HS}$, and young $\mathrm{PH}$ groups.

h Indicates significant $(\mathrm{P} \leq 0.05)$ difference from young HS group.

NMJs residing on those fibers. Whereas NMJs exclusively demonstrated significant main effects of aging, myofibers consistently manifested significant main effects not only for aging, but also for treatment as well as significant interactive effects. This was true both for myofiber cross-sectional area (size), and fiber type composition.

We first examined myofiber size without regard for fiber type, i.e., all fiber types pooled together. As expected given the known effects of sarcopenia, aged fibers were significantly smaller than the young adult myofibers, but post-hoc analysis indicated that while this aging effect was apparent in control and HS treatment groups, it was not noted among $\mathrm{PH}-\mathrm{HS}$, or $\mathrm{PH}$ treatment groups. Further scrutiny established that despite aged CTL animals having smaller myofibers than young CTLs, the amount of unloading-induced atrophy displayed by aged HS muscles was significantly more severe than that observed among young HS muscles ( $45 \%$ vs. $22 \%$, respectively). Interestingly, post-hoc analysis also documented that among young rats pre-habilitative exercise performed prior to muscle unloading actually significantly exacerbated unloading-related atrophy ( $40 \%$ vs. $22 \%$ in young $\mathrm{PH}-$ HS compared to young HS). In contrast, among aged rats the pre-habilitative exercise regimen significantly attenuated the atrophy elicited by unloading. More specifically, aged HS fibers experienced a $45 \%$ decline in fiber cross-sectional area whereas aged PH-HS myofibers evinced a $32 \%$ loss of size. Moreover, among young rats pre-habilitative conditioning by itself did not alter myofiber size, but in aged soleus muscles the same exercise program resulted in a significant increase (14\%) in myofiber size compared to age-specific CTL animals.

To gain further insight, we also examined the effects of age and treatment on individual myofiber types. Results from the ANOVA analysis of Type I fibers - which comprise the majority of soleus myofibers - revealed that aged CTLs were significantly smaller $(\sim 12 \%)$ than the Type I myofibers of young CTL animals. And similar to the data gathered with fiber types combined, unloading alone resulted in a significantly greater degree of atrophy among aged fibers than young ones ( $45 \%$ vs. $21 \%$ ). And again much like what was detected with fiber types collapsed together, Type I fibers showed that when unloading was preceded by exercise training, young myofibers exhibited a significantly greater degree of atrophy compared to unloading by itself (39\% vs. $21 \%$ ). Among aged Type I fibers, however, pre-habilitative conditioning significantly modulated the atrophy of unloading ( $\mathrm{PH}-\mathrm{HS}=31 \%, \mathrm{HS}=45 \%$ ). And again as with fiber types combined, young Type I myofibers did not alter their size in response to pre-habilitative exercise by itself, while aged Type I fibers increased their cross-sectional area by $14 \%(P \leq 0.05)$ in response to the same exercise stimulus.

Type IIA myofibers also displayed the atrophic effect of aging as aged CTL fibers were significantly smaller $(\sim 42 \%)$ than young CTL Type IIA fibers. But unlike the Type I myofibers, young and aged Type IIA fibers showed an almost identical reduction in size as a result of unloading ( $29 \%$ vs. $28 \%$, respectively). But as with Type I fibers, pre-habilitative conditioning actually amplified the atrophic response to unloading in young soleus Type IIA myofibers. In contrast to Type I fibers, however, pre-habilitation failed to mitigate unloading-induced atrophy among aged Type IIA fibers. And although pre-habilitative training did not affect the size of young Type IIA fibers, it evoked a significant expansion in the cross-sectional areas of aged Type IIA fibers. These were the same age specific results detected among the majority Type I fibers of the soleus.

In assessing Type IIX fibers exclusively, aging was associated with a significant decrease $(\sim 25 \%)$ in the size of control myofibers. Moreover, unloading brought about a 33\% decline in the size of aged Type IIX fibers, which contrasts with young Type IIX fibers which experienced no significant atrophy among the HS group. Still, by combining pre-habilitation with unloading, young IIX myofibers demonstrated a $36 \%$ diminution in a cross-sectional area $(P \leq 0.05)$. And unlike the other fiber types of aged muscles, pre-habilitative exercise did not ameliorate unloading-induced atrophy among aged Type IIX myofibers. Consistent with the other fiber types examined, however, pre-habilitative training by itself resulted in a significant increase in the size of Type IIX fibers in aged soleus muscles, whereas it had no impact on young Type IIX myofibers.

In evaluating the effects of aging and treatment on fiber type composition, we noted that aged muscles had a significantly greater percentage of Type I fibers than the young ones. This was discerned in each of the four treatment groups. Yet in quantifying the impact of the intervention regimens - $\mathrm{HS}, \mathrm{PH}-\mathrm{HS}, \mathrm{PH}-$ on the percentage of Type I fibers, our data show that those interventions were unable to alter the percentage of that fiber type in either young, or aged soleus 
muscles. Similar results were found among Type IIA and IIX fibers in the aged soleus muscles. That is, none of the three intervention protocols employed elicited changes in the percentage of the subcategories of fast-twitch (Type II) fibers in aged muscles. But among young adult muscles, our findings revealed that unloading resulted in a greater percentage of Type IIA fibers compared to the three other treatment groups. This was accompanied by a smaller percentage of Type IIX fibers among those same aged HS soleus muscles. All data regarding myofiber profiles - cross-sectional area and fiber type composition - are presented in Table 4.

\section{Discussion}

Rehabilitative exercise regimens are routinely used by clinicians following a period of neuromuscular disuse to expedite recovery from the deleterious adaptations brought about by that condition. However, the potential of pre-habilitative conditioning - exercise performed prior to disuse - as a palliative measure to counter or minimize the negative adaptations associated with disuse is not well understood, nor is it commonly implemented. This is curious because in many circumstances (e.g. orthopedic surgery) it is known for some time in advance that the neuromuscular system will be subjected to an extended interval of disuse. It is important that clinicians understand how exercise conditioning performed before the enforced period of neuromuscular disuse may be utilized to limit, if not eliminate, the maladaptive neuromuscular remodeling typically observed following disuse.

In the present study, the effects of muscle unloading - such as that brought on during bed rest, or as crutches/wheel chair assisted ambulation - was employed to carefully assess the impact of pre-habilitation on the more commonly encountered partial disuse as opposed to the more severe total neuromuscular disuse (i.e., denervation, paralysis). Moreover, because the aged are more likely to be subjected to imposed neuromuscular disuse than young individuals as a result of an increased

Table 4

Effects of hindlimb suspension (HS), hindlimb suspension preceded by pre-habilitation (PH-HS), and pre-habilitation by itself (HS) on myofiber profiles of young and aged soleus muscles.

\begin{tabular}{|c|c|c|c|c|}
\hline $\begin{array}{l}\text { Cross-sectional area } \\
\left(\mu \mathrm{m}^{2}\right)\end{array}$ & Control & HS & PH-HS & $\mathrm{PH}$ \\
\hline \multicolumn{5}{|l|}{ Young } \\
\hline Fiber types combined & $2518 \pm 75^{\mathrm{a}}$ & $1968 \pm 83^{\mathrm{ab}}$ & $1522 \pm 73^{b}$ & $2581 \pm 112$ \\
\hline Type I & $2513 \pm 80^{\mathrm{a}}$ & $1975 \pm 85^{\mathrm{ab}}$ & $1540 \pm 76^{\mathrm{b}}$ & $2534 \pm 125$ \\
\hline Type IIA & $2802 \pm 105^{a}$ & $1984 \pm 111^{\mathrm{ab}}$ & $1413 \pm 45^{\mathrm{b}}$ & $2754 \pm 174^{\mathrm{a}}$ \\
\hline Type IIX & $2053 \pm 174^{\mathrm{a}}$ & $1756 \pm 117^{\mathrm{ac}}$ & $1315 \pm 86^{\mathrm{b}}$ & $2314 \pm 184$ \\
\hline \multicolumn{5}{|c|}{ Fiber type composition (\%) } \\
\hline Type I & $88.7 \pm 1.2^{\mathrm{ad}}$ & $84.4 \pm 1.5^{\mathrm{a}}$ & $85.5 \pm 2.1^{\mathrm{a}}$ & $86.7 \pm 1.3^{\mathrm{a}}$ \\
\hline Type IIA & $7.0 \pm 1.0$ & $12.6 \pm 1.6^{\mathrm{ab}}$ & $7.5 \pm 1.5^{\mathrm{a}}$ & $6.5 \pm 1.3$ \\
\hline Type IIX & $4.3 \pm 0.5$ & $3.0 \pm 0.7^{c}$ & $7.0 \pm 1.5^{\mathrm{a}}$ & $6.8 \pm 1.3^{\mathrm{a}}$ \\
\hline \multicolumn{5}{|l|}{ Aged } \\
\hline Fiber types combined & $2182 \pm 71^{\mathrm{b}}$ & $1203 \pm 76^{\mathrm{b}}$ & $1492 \pm 101^{\mathrm{b}}$ & $2491 \pm 101^{\mathrm{b}}$ \\
\hline Type I & $2203 \pm 84^{b}$ & $1203 \pm 80^{\mathrm{b}}$ & $1513 \pm 105^{\mathrm{b}}$ & $2524 \pm 106^{\mathrm{b}}$ \\
\hline Type IIA & $1623 \pm 152^{c}$ & $1170 \pm 95$ & $1113 \pm 84$ & $2102 \pm 157^{c}$ \\
\hline Type IIX & $1531 \pm 121^{\mathrm{b}}$ & $1031 \pm 68$ & $1140 \pm 88$ & $2055 \pm 187^{c}$ \\
\hline \multicolumn{5}{|c|}{ Fiber type composition (\%) } \\
\hline Type I & $92.6 \pm 1.6$ & $91.8 \pm 1.6$ & $94.9 \pm 0.9$ & $92.4 \pm 1.7$ \\
\hline Type IIA & $4.7 \pm 1.3$ & $5.2 \pm 1.4$ & $2.4 \pm 0.5$ & $4.3 \pm 1.4$ \\
\hline Type IIX & $2.7 \pm 0.5$ & $3.0 \pm 0.7$ & $2.7 \pm 0.5$ & $3.3 \pm 0.8$ \\
\hline
\end{tabular}

Values are means $\pm \mathrm{SE}, \mathrm{N}=10$ /group

${ }^{a}$ Indicates significant $(\mathrm{P} \leq 0.05)$ difference from aged of the same treatment group and fiber type.

${ }^{b}$ Indicates significant $(\mathrm{P} \leq 0.05)$ difference from all other treatment groups of the same age and fiber type.

${ }^{c}$ Indicates significant $(\mathrm{P} \leq 0.05)$ difference from the $\mathrm{PH}-\mathrm{HS}$, and $\mathrm{PH}$ treatment groups of the same age and fiber type.

${ }^{d}$ Indicates significant $(P \leq 0.05)$ difference from the HS group of the same age and fiber type. incidence of accidental falls and surgery (Lloyd et al., 2009; Scott et al., 2011), we sought to establish whether aging influenced the efficacy of pre-habilitative conditioning. And to further expand our understanding of neuromuscular responsiveness to pre-habilitation, we quantified not only the changes in myofiber profiles to unloading with, and without pre-habilitation, but also those of the NMJs that are responsible for neural communication with those myofibers. Our analyses generated a number of interesting and potentially useful clinical findings. We first examined NMJs and it was determined both in young adult and aged muscles that 2 weeks of muscle unloading was insufficient to elicit significant synaptic remodeling. This should be considered in light of earlier reports documenting that 4 weeks of the same model of disuse - muscle unloading evoked by hindlimb suspension resulted in significant remodeling of aged, but not young NMJs (Deschenes and Wilson, 2003), but that 10 weeks of hindlimb suspension elicits significant reconfiguration of the NMJ even among young muscles (Deschenes et al., 2006). Clearly then, aging does affect the responsiveness of the NMJ to incomplete neuromuscular disuse, but a two week intervention period is insufficient even among the aged to bring about synaptic modifications of the peripheral nervous system.

In contrast to the lack of responsiveness to the main effect of treatment, the main effect of aging was associated with a myriad of significant adaptations that were identified both in the pre- and post-synaptic components of the NMJ. In general, aging was characterized by an expansion of pre-synaptic nerve terminal branching, with a decreased area of the post-synaptic endplate occupied by ACh receptors which featured a more dispersed distribution of those receptors. The combination of these pre- and post-synaptic alterations resulted in a modified architectural relationship between the ACh release sites of nerve terminal branches and their post-synaptic ACh binding sites. More specifically, aged synapses appear to have fewer post-synaptic binding sites (receptors) for a given segment of the pre-synaptic branches that anchor ACh release sites (vesicles). Some, but not all, of the age-related morphological adaptations noted here have been reported elsewhere (Anis and Robbins, 1987; Fahim et al., 1983; Pestronk and Drachman, 1985; Pestronk et al., 1980; Robbins and Fahim, 1985).

A particularly interesting, and novel, finding of the present investigation was made possible by our ability to not only quantify pre- and post-synaptic components of the NMJ, but also to determine whether the NMJ in question was located on a slow- or a fast-twitch myofiber. The value of this stems from the well-known differences in recruitment patterns, and thus normal activity levels, between slow- and fast-twitch myofibers. Recall that slow motor units featuring slow-twitch myofibers are more easily recruited and display higher rates of activity than fast motor units and their fast-twitch myofibers (Luscher et al., 1979; Senn et al., 1997). The data presented here establish that both slow- and fast-twitch NMJs exhibited age-related remodeling, but it was the fast-twitch ones that evinced this across all 10 parameters assessed, while among slow-twitch NMJs only six of the same 10 variables demonstrated significant effects of aging. This fiber-type specificity in age-related NMJ adaptation is probably explained by the fact that slow-twitch myofibers remain more active than the fast-twitch ones and, accordingly, are more resilient to age-related structural changes. Indeed, there is compelling evidence that regular physical activity moderates adaptations to senescence in many physiological systems of the body (Ahlskog et al., 2011; Allen and Morelli, 2011; Haaland et al., 2008; Hart et al., 2008; Kappagoda and Amsterdam, 2012; Woods et al., 2012).

Unlike NMJs, where only significant main effects of age were evident, our analysis of myofiber profiles consistently identified not only the main effects for both age and treatment, but also for interaction thus confirming that aged and young adult myofibers responded differently to the interventions of unloading, unloading preceded by pre-habilitation, and pre-habilitative exercise by itself. As expected given the well documented effects of sarcopenia (Deschenes, 2004; 

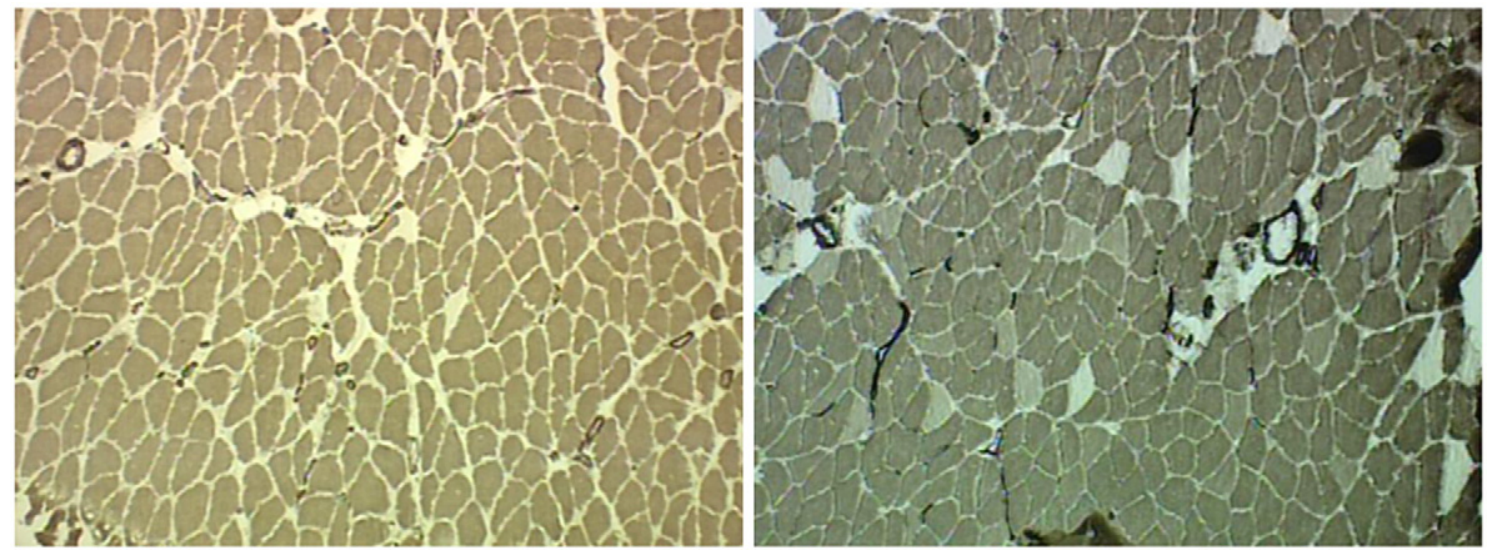

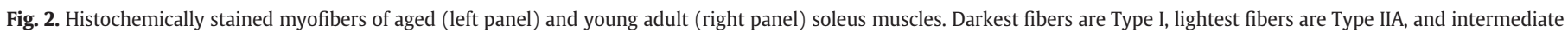
stained fibers are Type IIX. Note the smaller fiber size and limited number of Type II fibers in the aged soleus. Original magnification of $100 \times$.

Frontera et al., 2012; Visser and Schaap, 2011), the myofibers of aged muscles were significantly smaller and featured a higher percentage of Type I fibers compared to young adult muscles (Fig. 2).

The present study re-affirmed what we previously reported (Deschenes et al., 2001) in that compared to young ones, aged myofibers suffer significantly more atrophy as a result of muscle unloading. Since it is known that aging already imparts an atrophic milieu among muscles - thus contributing to sarcopenia - it is reasonable, if not demonstrable, that the disuse of unloading serves to exacerbate that naturally occurring atrophic trend and that it is this increasingly atrophic environment that explains why aged muscles are more susceptible to the muscle wasting response of muscle unloading. Similarly, it is possible that the acceleration of the naturally occurring process of sarcopenia brought about by unloading may account for the fact that the pre-habilitative regimen significantly attenuated the unloading-induced atrophy noted among aged, but not young muscles. That is, the pre-habilitative exercise performed by the aged prior to unloading may have at least partially neutralized, or interfered with the progress of sarcopenia by triggering the hypertrophic pathways which were able to retard the rapid rate of unloading-induced atrophy among aged myofibers.

The fact that when conducted by itself, pre-habilitative exercise was accompanied by significant hypertrophy in aged, but not young muscles can be viewed as supportive evidence of this proposition that once myofibers enter the pathway to sarcopenic-atrophy they may actually be more sensitive to the hypertrophic stimulus of exercise. Moreover, it should not be overlooked that among young adult muscles - those not undergoing the atrophic processes of sarcopenia - the type of endurance training used here during pre-habilitative conditioning typically results in moderate $(\sim 10 \%)$ but significant decreases in myofiber size. This is in contrast with aged, sarcopenic myofibers where that same exercise stimulus was responsible for a hypertrophic, rather than an atrophic effect, both here and in an earlier report (Brown et al., 1992). The conflicting outcomes of endurance training on young and aged myofibers lend credibility to the assertion that the combined atrophic stimuli of sarcopenia and muscle unloading occurring in aged muscle may actually enhance the sensitivity of those fibers to the hypertrophic effects of exercise.

In assessing the compiled data gathered from NMJs and myofibers, a quandary that arises relates to the fact that both young adult and aged myofibers were responsive to the various treatment interventions, but the NMJs of those fibers remained resistant to those same interventions. A reasonable explanation to this relates to the fact that the NMJ and the contractile apparatus of myofibers have vastly different principal functions resulting in divergent sensitivities to muscle unloading. Using electromyography, Alford et al. (1987) have shown that unloading of the soleus alters the nerve to muscle communication for no more than
2 days, confirming that the main function of the NMJ is scarcely affected as a result of unloading. This differs from the other forms of disuse such as denervation or the application of neurotoxin where extensive remodeling of the NMJ is apparent even in a short time frame (Brown and Ironton, 1978; Pachter and Spielholz, 1990; Pestronk and Drachman, 1978; Tsujimoto and Kuno, 1988). In contrast to the NMJ however, muscle unloading - particularly of the weight bearing soleus - dramatically disturbs the normal contractile and force generating function of the myofibers' contractile apparatus resulting in equally dramatic remodeling of those same myofibers whose NMJs are resilient to the same intervention of muscle unloading.

In summary, our investigation testing the capacity of pre-habilitative activity to mitigate the detrimental neuromuscular effects of muscle unloading produced mixed outcomes. First, our two week period of incomplete disuse as presented by muscle unloading was not potent enough to elicit adaptations within the NMJ so the prophylactic impact of pre-habilitation could not be deduced at the synaptic level. But when examining the impact of unloading on the myofiber size, it was revealed that this same model of incomplete disuse led to significant amounts of atrophy in both young and aged muscles, with the latter demonstrating a significantly greater degree of muscle wasting than the former. Importantly, it was also found that it was only among aged muscles - which were more sensitive to the atrophic stimulus of unloading - that pre-habilitative conditioning was able to successfully ameliorate unloading-induced atrophy. This information should be useful for clinicians since it is among the aged that the intervention of imposed muscle unloading most commonly occurs and it is among that same population that the atrophic adaptations to unloading are most severe.

\section{Acknowledgments}

This work was supported by grants from the National Institutes of Health (R15 AG17440), and the Borgenicht Program for Aging Studies and Exercise Science.

\section{References}

Adams, G.R., Caiozzo, V.J., Baldwin, K.M., 2003. Skeletal muscle unweighting: spaceflight and ground-based models. J. Appl. Physiol. 95, 2185-2201.

Ahlskog, J.E., Geda, Y.E., Graff-Radford, N.R., Petersen, R.C., 2011. Physical exercise as a preventive or disease-modifying treatment of dementia and brain aging. Mayo Clin. Proc. 86, 876-884.

Alford, E.K., Roy, R.R., Hodgson, J.A., Edgerton, V.R., 1987. Electromyography of rat soleus, medial gastrocnemius, and tibialis anterior during hind limb suspension. Exp. Neurol. 96, 635-649.

Allen, J., Morelli, V., 2011. Aging and exercise. Clin. Geriatr. Med. 27, 661-671.

Anderton, B.H., Breinburg, D., Downes, M.J., Green, P.J., Tomlinson, B.E., Ulrich, J., Wood, J.N., Kahn, J., 1982. Monoclonal antibodies show that neurofibrillary tangles and neurofilaments share antigenic determinants. Nature 298, 84-86. 
Anis, N.A., Robbins, N., 1987. General and strain-specific age changes at mouse limb neuromuscular junctions. Neurobiol. Aging 8, 309-318.

Arias, E., 2006. United States life tables, 2003. Natl. Vital Stat. Rep. 54, 1-40.

Armstrong, R.B., Phelps, R.O., 1984. Muscle fiber type composition of the rat hindlimb. Am. J. Anat. 171, 259-272.

Brown, M.C., Ironton, R., 1978. Sprouting and regression of neuromuscular synapses in partially denervated mammalian muscles. J. Physiol. 278, 325-348.

Brown, M., Ross, T.P., Holloszy, J.O., 1992. Effects of ageing and exercise on soleus and extensor digitorum longus muscles of female rats. Mech. Ageing Dev. 63, 69-77.

Caiozzo, V.J., Haddad, F., Baker, M.J., Baldwin, K.M., 1995. Functional and cellular adaptations of rodent skeletal muscle to weightlessness. J. Gravit. Physiol. 2, P39-P42.

DeLorme, T.L., Watkins, A.L., 1948. Technics of progressive resistance exercise. Arch. Phys. Med. Rehabil. 29, 263-273.

DeLorme, T.L., West, F.E., Shriber, W.J., 1950. Influence of progressive resistance exercises on knee function following femoral fractures. J. Bone Joint Surg. Am. 32, 910-924.

Delp, M.D., Duan, C., 1996. Composition and size of type I, IIA, IID/X, and IIB fibers and citrate synthase activity of rat muscle. J. Appl. Physiol. 80, 261-270.

Deschenes, M.R., 2004. Effects of aging on muscle fibre type and size. Sports Med. 34, 809-824.

Deschenes, M.R., Wilson, M.H., 2003. Age-related differences in synaptic plasticity following muscle unloading. J. Neurobiol. 57, 246-256.

Deschenes, M.R., Britt, A.A., Chandler, W.C., 2001. A comparison of the effects of unloading in young adult and aged skeletal muscle. Med. Sci. Sports Exerc. 33, 1477-1483.

Deschenes, M.R., Wilson, M.H., Kraemer, W.J., 2005. Neuromuscular adaptations to spaceflight are specific to postural muscles. Muscle Nerve 31, 468-474.

Deschenes, M.R., Tenny, K.A., Wilson, M.H., 2006. Increased and decreased activity elicits specific morphological adaptations of the neuromuscular junction. Neuroscience 137, 1277-1283.

Fahim, M.A., Holley, J.A., Robbins, N., 1983. Scanning and light microscopic study of age changes at a neuromuscular junction in the mouse. J. Neurocytol. 12, 13-25.

Fitts, R.H., Riley, D.R., Widrick, J.J., 2000. Physiology of a microgravity environment invited review: microgravity and skeletal muscle. J. Appl. Physiol. 89, 823-839.

Frontera, W.R., Zayas, A.R., Rodriguez, N., 2012. Aging of human muscle: understanding sarcopenia at the single muscle cell level. Phys. Med. Rehabil. Clin. N. Am. 23, 201-207 (xiii).

Haaland, D.A., Sabljic, T.F., Baribeau, D.A., Mukovozov, I.M., Hart, L.E., 2008. Is regular exercise a friend or foe of the aging immune system? A systematic review. Clin. J. Sport Med. 18, 539-548.

Hart, L.E., Haaland, D.A., Baribeau, D.A., Mukovozov, I.M., Sabljic, T.F., 2008. The relationship between exercise and osteoarthritis in the elderly. Clin. J. Sport Med. 18, 508-521.

Kappagoda, T., Amsterdam, E.A., 2012. Exercise and heart failure in the elderly. Heart Fail. Rev. 17, 635-662.

Kraemer, W.J., Staron, R.S., Gordon, S.E., Volek, J.S., Koziris, L.P., Duncan, N.D., Nindl, B.C., Gomez, A.L., Marx, J.O., Fry, A.C., Murray, J.D., 2000. The effects of 10 days of spaceflight on the shuttle endeavor on predominantly fast-twitch muscles in the rat. Histochem. Cell Biol. 114, 349-355.

Lavernia, C., Lee, D.J., Hernandez, V.H., 2006. The increasing financial burden of knee revision surgery in the United States. Clin. Orthop. Relat. Res. 446, 221-226.

Lipsitz, L.A., Nakajima, I., Gagnon, M., Hirayama, T., Connelly, C.M., Izumo, H., Hirayama, T., 1994. Muscle strength and fall rates among residents of Japanese and American nursing homes: an international cross-cultural study. J. Am. Geriatr. Soc. 42, 953-959.
Lloyd, B.D., Williamson, D.A., Singh, N.A., Hansen, R.D., Diamond, T.H., Finnegan, T.P., Allen, B.J., Grady, J.N., Stavrinos, T.M., Smith, E.U., Diwan, A.D., Fiatarone Singh, M.A., 2009. Recurrent and injurious falls in the year following hip fracture: a prospective study of incidence and risk factors from the sarcopenia and hip fracture study. J. Gerontol. A Biol. Sci. Med. Sci. 64, 599-609.

Luscher, H.R., Ruenzel, P., Henneman, E., 1979. How the size of motoneurones determines their susceptibility to discharge. Nature 282, 859-861.

Morey, E.R., 1979. Spaceflight and bone turnover: correlation with a new rat model of weightlessness. Bioscience 29, 168-172.

Musacchia, X.J., Steffen, J.M., Fell, R.D., Dombrowski, M.J., 1990. Skeletal muscle response to spaceflight, whole body suspension, and recovery in rats. J. Appl. Physiol. 69, 2248-2253.

Nemeth, P., Pette, D., 1981. Succinate dehydrogenase activity in fibres classified by myosin ATPase in three hind limb muscles of rat. J. Physiol. 320, 73-80.

Pachter, B.R., Spielholz, N.I., 1990. Tenotomy-induced motor endplate alterations in rat soleus muscle. Anat. Rec. 228, 104-108.

Pearson, J., Sabarra, A., 1974. A method for obtaining longitudinal cryostat sections of living muscle without contraction artifacts. Stain. Technol. 49, 143-146.

Pestronk, A., Drachman, D.B., 1978. Motor nerve sprouting and acetylcholine receptors. Science 199, 1223-1225.

Pestronk, A., Drachman, D.B., 1985. Motor nerve terminal outgrowth and acetylcholine receptors: inhibition of terminal outgrowth by alpha-bungarotoxin and anti-ace tylcholine receptor antibody. J. Neurosci. 5, 751-758.

Pestronk, A., Drachman, D.B., Griffin, J.W., 1980. Effects of aging on nerve sprouting and regeneration. Exp. Neurol. 70, 65-82.

Robbins, N., Fahim, M.A., 1985. Progression of age changes in mature mouse motor nerve terminals and its relation to locomotor activity. J. Neurocytol. 14, 1019-1036.

Roy, R.R., Hirota, W.K., Kuehl, M., Edgerton, V.R., 1985. Recruitment patterns in the rat hindlimb muscle during swimming. Brain Res. 337, 175-178.

Roy, R.R., Hutchison, D.L., Pierotti, D.J., Hodgson, J.A., Edgerton, V.R., 1991. EMG patterns of rat ankle extensors and flexors during treadmill locomotion and swimming. J Appl. Physiol. 70, 2522-2529.

Scott, D., Blizzard, L., Fell, J., Jones, G., 2011. The epidemiology of sarcopenia in community living older adults: what role does lifestyle play? J. Cachexia Sarcopenia Muscle 2, 125-134.

Senn, W., Wyler, K., Clamann, H.P., Kleinle, J., Luscher, H.R., Muller, L., 1997. Size principle and information theory. Biol. Cybern. 76, 11-22.

Tomas, J., Fenoll, R., Mayayo, E., Santafe, M., 1990. Branching pattern of the motor nerve endings in a skeletal muscle of the adult rat. J. Anat. 168, 123-135.

Tsujimoto, T., Kuno, M., 1988. Calcitonin gene-related peptide prevents disuse-induced sprouting of rat motor nerve terminals. J. Neurosci. 8, 3951-3957.

Turturro, A., Witt, W.W., Lewis, S., Hass, B.S., Lipman, R.D., Hart, R.W., 1999. Growth curves and survival characteristics of animals used in the biomarkers of aging program. J. Gerontol. A Biol. Sci. Med. Sci. 54, B492-B501.

Visser, M., Schaap, L.A., 2011. Consequences of sarcopenia. Clin. Geriatr. Med. 27, 387-399.

Woods, J.A., Wilund, K.R., Martin, S.A., Kistler, B.M., 2012. Exercise, inflammation and aging. Aging Dis. 3, 130-140. 